\title{
Scope for optimal Scheduling of Multiproduct Pipelines with Multi-batch Sequential transportation
}

\author{
Qi Liaol, Yongtu Liang ${ }^{1 \star}$, Huixia Feng ${ }^{1}$, Zhengbing $\mathrm{Li}^{1}$ and Haoran Zhang ${ }^{2 \star}$ \\ ${ }^{I}$ China University of Petroleum-Beijing, Beijing Key Laboratory of Urban oil and Gas Distribution Technology, Fuxue Road No. 18, \\ Changping District, Beijing, China \\ ${ }^{2}$ Center for Spatial Information Science, The University of Tokyo 5-1-5 Kashiwanoha, Kashiwa-shi, Chiba, Japan
}

\section{Article Info}

*Corresponding authors:
Yongtu Liang
China University of Petroleum-Beijing
Beijing Key Laboratory of Urban oil and
Gas Distribution Technology
Fuxue Road No.18, Changping District
Beijing, China
E-mail: liangyt21st@163.com

\section{Haoran Zhang}

Center for Spatial Information Science

The University of Tokyo 5-1-5 Kashiwanoha Kashiwa-shi, Chiba, Japan

E-mail: zhang_ronan@csis.u-tokyo.ac.jp

Received: September 20, 2018
Accepted: November 23, 2018
Published: November 28, 2018

Citation: Liao $Q$, Liang $Y$, Feng $H$, Li Z, Zhang $\mathrm{H}$. Scope for optimal Scheduling of Multiproduct Pipelines with Multi-batch Sequential transportation. Int I Petrochem Res. 2018; 2(3): 202-206.

doi: 10.18689/ijpr-1000136

Copyright: @ 2018 The Author(s). This work is licensed under a Creative Commons Attribution 4.0 International License, which permits unrestricted use, distribution, and reproduction in any medium, provided the original work is properly cited.

Published by Madridge Publishers

\begin{abstract}
The optimal scheduling of multiproduct pipelines is benefit to improve the transport economy and safety, thus making great sense to the operation and management of multiproduct pipelines. During the last decade, a large number of domestic and foreign studies have been widely done on this topic. Based on the related literature, the paper summarizes the research emphasis, analyzes different modeling methods and solving algorithms 4Kand finally provides the outlook for the further researches on the scheduling optimization.
\end{abstract}

Keywords: Multiproduct pipeline; Scheduling optimization; Modeling method; Solving algorithm

\section{Introduction}

The multiproduct pipeline is a complex and large-scale system composed of refineries, injection stations, delivery stations, pump stations, storage tanks and the pipelines connected with it. The pipeline operators should take downstream market demand, refinery production capacity, conveying capacity, and storage profiles along the pipeline into account to rationally allocate resources and work out the detailed scheduling plan [1]. Meanwhile, the scheduling plan is evaluated and verified from the angle of the production safety and feasibility, so as to reach the goals, such as the minimum make spa [2-4], minimal operational cost $[5,6]$, the most stable operating condition [7] and the highest market satisfaction [8]. In addition, since the operations of multiproduct pipelines are affected by downstream market demand, the scheduling plans need to be modified continuously during the execution to meet the changing market demand and transportation technology [9]. Therefore, it makes sense for pipeline management to quickly draw the economic and feasible scheduling plans according to the fluctuating market environment and production environment. This paper investigates the recent works on the optimal scheduling of multiproduct pipelines, summarizes the emphasis, dissects the different modeling methods, summarizes the common solving strategies and finally puts forward the future prospect based on the research status.

\section{Objectives}

The scheduling optimization of multiproduct pipelines generally involves the following optimization sub-problems: batch sequenceand lot-sizing, injection and delivery operations, pump operations, inventory management and contaminated product control. These sub-problems, involving a large number of factors and even some nonlinear and uncertain items are coupled with each other, thus making it 
challenging to deal with the scheduling optimization $[10,11]$. At the same time, complex pipeline structure and long scheduling horizon would greatly increase the model scale, resulting in high computational effort $[5,12]$. This section summarizes the recent-year research on pipeline scheduling optimization based on following optimization objectives.

\section{Energy consumption}

As time goes on, the characteristic curves of pump as well as pipeline continuously change with batch migration, causing the change in the working point of the pump. To keep the pipeline working within the allowable pressure range, a set of measures for adjusting the operating condition are provided based on conservation of energy, including starting (stopping) pumps and adjusting pump speed (delivery flowrate/ throttling valves). Hence, flexible operating conditions and highly nonlinear hydraulic calculation complicate the scheduling optimization accounting for energy consumption. The common method is to transform the complex hydraulic constraints into the corresponding flow constraints, and linearize the nonlinear terms associated with flowrate. For example, Abbasi and Garousi [13] assumes that the frictional loss and pump head are linear with flowrate since the flowrate is generally limited within a small range in practical engineering due to operational constraints. Moreover, piecewise linear approximation $[6,14]$, in which the flowrate is divided into several small ranges and the $X^{\text {th }}$ power of flowrate within each range can be approximately linearized, is widely used to deal with the nonlinear constraints introduced by flowrate. Apart from the above linearization methods, Cafaro, Cafaro [15] developed a novel MINLP model rigorously accounting for nonlinear formulas of energy consumption, using GAMSDICOPT as the MINLP solver. In addition, Chen, Zuo [7] draw a conclusion that stable pipeline flow is conducive to reducing frictional loss within the scheduling horizon, thus reducing the energy consumption of pumps. Afterward, they established a discrete-time MILP model with the minimum fluctuation of pipeline flow as the objective function, and proposed a metaheuristic algorithm based on simulated annealing [16] that greatly improved the solving efficiency.

\section{Contamination loss}

When multiple batches are conveyed without separation devices, the contaminated products are inevitably generated between any two adjacent batches under the diffusion action of convection and turbulent. The tracking and control of contaminated products is one of the key technologies of multiproduct pipelines. However, the characteristic, technical process and operation management related with contaminated products are complicated, especially in the area with complex topography and large elevation difference. Generally, the generated contaminated products cannot be sell off as qualified products and need to be processed by blending or refinery, which brings extra operational cost for petroleum companies [17]. Most of the previous work control the development of contaminated products from two aspects, one is to optimize batch sequencing [18-21], and the other is to add processing constraints in mathematical models. For example, aiming at batch sequencing and detailed scheduling of branch pipeline networks, Mostafaei, Castro [18] presented a unified optimization model with considering different processing cost depend on the contamination type and volume. Furthermore, to ensure product quality, forbidden batch sequence is also taken into account to prevent the products with excessive difference in physical property to be conveyed adjacently. With regarding the time nodes of batches just arriving at stations as the key time nodes, Zhang, Liang [8] developed a continuoustime MILP model and introduces two kinds of constraints to control the development of contaminated products: (a) More rigorous lower limits are imposed on the flowrate in the pipeline segments with contaminated products; (b) Shut-down operation should be forbidden as far as possible in the inclined pipeline segments especially when heavy oil is upper and light oil is lower. Meanwhile, Zhang, Liang [17] transformed nonlinear growth of the contaminated products into linearized Austin's formula based on piecewise linear approximation, and then established a mixed-time MILP model for the most low-cost operations of oil depots along a multiproduct pipeline.

\section{Market satisfaction}

Most of previous work set equality constraints for the products diverted at every station to satisfy the aggregate demand plan $[15,23]$. However, since the demand for products is separately proposed by each delivery station, the delivery stations may not receive the required volume of products under all the operational constraints. Considering that the feasible solution may not exist under these equality constraints, a part of studies developed MILP models with target of minimizing operational cost caused by the deviation between the actual received volume $\left(V x_{j, i}\right)$ and planned volume $\left(v_{j, i}\right)$ as the objective function, which is stated in Eq.(1) $[8,23,24]$. A series of artificial variables (i.e., $M 1_{j, i}$ and $M 2_{j, i}$ in Eq.(2)) are introduced to transform the nonlinear objective function Eq.(1) into linear Eq.(2). It should be noted that the unit cost $\left(d c_{j, i}^{m c}\right)$ comes in two forms, namely $d c_{j, i}^{1}$ and $d c_{j, i}^{2}$.Specifically, when the actual received volume is less than the planned volume, it is necessary to transport the products from other oil depot by railway or highway, which increases transportation cost. Instead, receiving extra products would bring additional cost of evaporation loss and inventory management.

$$
\begin{aligned}
& \min f=\sum_{j \in J} \sum_{i \in I} d c_{j, i}^{m c}\left|V x_{j, i}-v s_{j, i}\right| \\
& \left\{\begin{array}{l}
m c=1 \text { if }\left(V x_{j, i}-v s_{j, i}\right) \geq 0, j \in J, i \in I \\
m c=2 \text { if }\left(V x_{j, i}-v s_{j, i}\right)<0, j \in J, i \in I
\end{array}\right. \\
& \min f=\sum_{j \in J} \sum_{i \in I}\left(d c_{j, i}^{1} M 1_{j, i}+d c_{j, i}^{2} M 2_{j, i}\right) \\
& \left\{\begin{array}{l}
M 1_{j, i} \geq V x_{j, i}-v s_{j, i}, j \in J, i \in I \\
M 2_{j, i} \geq v s_{j, i}-V x_{j, i}, j \in J, i \in I
\end{array}\right.
\end{aligned}
$$

\section{Inventory management}

The detailed scheduling plan should be made with full consideration of storage profiles along the pipeline, the coordination between oil sources and oil depots, As well as the 
batches pre existing in each pipeline segment [25]. The operation times of oil tanks should also be reduced as far as possible to minimize the oil loss during transportation process. Besides, the liquid level of all the tanks should be with allowable range when receiving or exporting products. Therefore, a large number of studies took the labor cost caused by switching oil tanks as the objective function, While the inventory limit as constraints to establish mathematical models for the optimal operations of tanks [26-28]. In addition, the transfer depot at the intersection among pipelines in the pipeline network can serve as the important adjustment method to cope with the fluctuation of supply and demand. In other words, excess products can be exported to transfer depots for storage when faced with over supply problem, whereas the products stored at transfer depots can be imported into the pipelines to supplement the shortage of products when faced with faced with insufficient supply. It can be concluded that the storage capacity of transfer depots largely determines the adjustment capacity of pipelines.

\section{Modeling method}

\section{Mathematical programming}

The mathematical programming method can simplify the problem into a mathematical programming model, the optimal solution of which can be found by integer programming, dynamic programming, decision analysis, etc. By this way, a large number of binary variables are introduced to describe the logical relationship among the variables. While when modeling for practical engineering, a set of nonlinear constraints cannot be ignored, thus increasing the modeling difficulty. Therefore, MILP models and MINLP models are widely used in the scheduling issues. Over the past decade, Cafaro et al, [29-32], Relvas et al. [33,34] and Mir Hassani et al. $[35,36]$ carried out extensive research on MILP models aiming at different time representatives and pipeline structure. Later on, some studies also presented MINLP models coupling with hydraulic calculation to ensure the safe and efficient operations of multiproduct pipelines. However, the MINLP model is usually a non-convex model, so it is difficult to find the optimal solution within the acceptable time range or even a feasible solution. To resolve this problem, some solution strategies gradually appear, such as decomposition algorithm, aggregation algorithm and heuristic algorithm. For instance, Rejowski and Pinto [37] and Cafaro, Cafaro [15] adopted the GAMS- DICOPT [38] to solve MINLP models accounting for pump energy consumption. By comprehensively considering constraints such as injection, delivery, batch migration, power consumption of pumps and time-dependent electricity price, Zhang, Liang [23] established a continuous-time MINLP model, then decomposed it into an integer programming (IP) problem and a linear programming problem(LP), and finally solved these two sub-problems iteratively to find the optimal or sub-optimal solution of the original problem.

\section{Generalized Disjunctive Programming (GDP)}

Although mathematical programming models can deal with most of the scheduling optimization problems, they usually contain a large number of binary variables and big- $M$ constraints, which result in weak linear relaxations and poor computational performance when faced with large-scale issue [39]. To avoid the above drawbacks, Raman and Grossmann [40] proposed a high-level framework based on equations and symbolic logic. Under such a framework, the model structure can be simplified by replacing previous binary variables with Boolean variables. In addition, the GDP model can use logical reasoning to eliminate the unfeasible solution domain, reduce the searching space, and improve the solution efficiency [41]. Mostafaei and Castro [22] relied on GDP to propose a continuous-time model for the detailed scheduling of multi-source and multi-sink pipelines, significantly improving the utilization rate of pipelines and shortening the make span. They inserted a number of empty batches to define new batches injected by the intermediate injection stations, resulting in a sharp increase in model size. Moreover, the model cannot strictly consider forbidden batch sequence when empty batch exists. Hence Castro and Mostafaei [3] extended their work a product-centric model based on GDP. Compared with a product-centric formulation based on the Resource-Task Network (RTN) [22], the new GDP-based one only needs roughly one quarter of the binary variables for the same linear relaxation, greatly reducing model scale and improving solving efficiency.

\section{Resource Task Network (RTN)}

RTN is a network composed of resource nodes and task nodes, among which resource nodes stand for all entities involved in the process steps, whereas task nodes stand for operations that transform a certain set of resources into another set $[42,43]$. A general RTN model is simple in concept and can be directly applicable to a very wide range of process scheduling systems. Therefore, RTN is adopted to establish a unified continuous-time or discrete-time framework for the scheduling of large-scale multiproduct pipeline system [44]. For example, Castro [45] regarded products, refineries, oil depots and pipelines as resource nodes, while injection, transport and delivery as task nodes, and then established a continuous time RTN model for the branched pipeline network. Although RTN can intuitively describe the transportation process of products, the construction of RTN becomes extremely complex when dealing with largescale issues containing complicating operational constraints.

\section{Reschedule}

Product pipeline scheduling is a continuous dynamic process involving a lot of uncertainties. Rescheduling is a common method to solve uncertain scheduling optimization, which is classified into passive scheduling and active scheduling. The former one is similar to the manual scheduling; that is to say, the prepared scheduling plan is adjusted or reformulated according to the real-time market situation, such as a reactive scheduling method proposed by Relvas, Matos [46]. The latter one is to adopt the stochastic optimization approach that explicitly model the uncertainties to generate the optimal scheduling plan. Common stochastic optimization methods include fuzzy programming [47], multiparameter methods $[48,49]$, simulation methods [50], robust 
optimization [51] and multi-stage stochastic programming $[52,53]$. Although the above stochastic optimization methods can consider the uncertainty of pipeline system comprehensively, they may lead to low efficiency or unsatisfactory solution. Overall, the passive scheduling model is generally applicable to the following conditions: (a) short-term scheduling problems with little uncertainty; (b) the requirement for computational efficiency is higher than the optimality of the scheduling plan. However, the active scheduling model is generally applied to solve the optimal scheduling plan when the scheduling horizon is long or the scheduling frequency is low [9].

\section{Conclusions and prospects}

The detailed scheduling of multiproduct pipeline has been one of the most challenging problems faced by pipeline operators. According to the existing literature, the current research mainly focuses on three aspects: (a) solving efficiency, that is, how to solve the scheduling optimization model of complex pipeline network efficiently to meet the timeliness of scheduling plan; (b) planning safety, that is, how hydraulic constraints are coupled in the model to meet the requirements of safe operation; (c) economy, such as how to consider the amount of contaminated products or pump energy consumption during the transportation process. In general, the following issues can be further discussed in the future.

(1) Existing studies usually adopt a decomposition strategy to optimize the pipeline scheduling plan and pump operation plan separately. Although this method can simplify the scale of the overall model and reduce computational time, the flow rate obtained by the first step may not be within the high-efficient interval of centrifugal pumps, thus affecting the economy of pump operation plan solved by the second step.

(2) How to strictly consider the hydraulic calculation that varies with batch migration, and establish the scheduling optimization model with the pressure constraint of key nodes to guarantee transportation safety.

(3) How to develop high-efficient approach based on a large amount of existing operational, deep learning and data mining.

(4) There are a lot of uncertain factors in the multiproduct pipeline network. The introduction of these factors will certainly increase the modeling difficulty. How to effectively combine fuzzy programming and probability methods to solve the pipeline scheduling optimization under uncertain conditions is still an important direction of future research.

\section{Acknowledgments}

This work was part of the Program of "Study on Optimization and Supply-side Reliability of Oil Product Supply Chain Logistics System" funded under the National Natural Science Foundation of China, grant number 51874325. The authors are grateful to all study participants.

\section{References}

1. Liao Q, Zhang H, Xu N, Liang Y, Wang J. A MILP model based on flowrate database for detailed scheduling of a multi-product pipeline with multiple pump stations. Computers \& Chemical Engineering. 2018; 117: 63-81. doi: 10.1016/j.compchemeng.2018.05.002

2. Castro PM. Optimal Scheduling of Multiproduct Pipelines in Networks with Reversible Flow. Ind. Eng. Chem. Res. 2017; 56(34): 9638-9656. doi: 10.1021/acs.iecr.7b01685

3. Castro PM, Mostafaei H. Product-centric continuous-time formulation for pipeline scheduling. Computers \& Chemical Engineering. 2017; 104: 283295. doi: 10.1016/j.compchemeng.2017.04.023

4. Cafaro DC, Cerdá J. Rigorous scheduling of mesh-structure refined petroleum pipeline networks. Computers \& Chemical Engineering. 2012; 38: 185-203. doi: 10.1016/j.compchemeng.2011.11.007

5. Mostafaei H, Ghaffari Hadigheh A. A General Modeling Framework for the Long-Term Scheduling of Multiproduct Pipelines with Delivery Constraints. Ind. Eng. Chem. Res. 2014; 53(17): 7029-7042. doi: 10.1021/ ie4038032doi

6. Liao Q, Liang $Y, X u N$, Zhang H, Wang J, Zhou X. An MILP approach for detailed scheduling of multi-product pipeline in pressure control mode. Chemical Engineering Research and Design. 2018; 136: 620-637. doi: 10.1016/j.cherd.2018.06.016

7. Chen $\mathrm{H}, \mathrm{Zuo} \mathrm{L}, \mathrm{Wu}$, et al. Optimizing detailed schedules of a multiproduct pipeline by a monolithic MILP formulation. Journal of Petroleum Science and Engineering. 2017; 159: 148-163. doi: 10.1016/j.petrol.2017.09.036

8. Zhang $H$, Liang $Y$, Liao $Q$, Shen $Y$, Yan X. A self-learning approach for optimal detailed scheduling of multi-product pipeline. Journal of Computational and Applied Mathematics. 2018; 327: 41-63. doi: 10.1016/j. cam.2017.05.040

9. Harjunkoski I, Maravelias CT, Bongers $P$, et al. Scope for industrial applications of production scheduling models and solution methods. Computers \& Chemical Engineering. 2014; 62: 161-193. doi: 10.1016/j. compchemeng.2013.12.001

10. Zhigang D, Yongtu L, Qiang G, Qiao X, Haoran Z, Guoxi H. An automatic detailed scheduling method of refined products pipeline. Conference An automatic detailed scheduling method of refined products pipeline. IEEE International Conference on Control and Automation (ICCA). 2016; 816-823.

11. Relvas S, Matos HA, Barbosa-Póvoa APFD, Fialho J, Pinheiro AS. Pipeline Scheduling and Inventory Management of a Multiproduct Distribution Oil System. Ind. Eng. Chem. Res. 2006; 45(23): 7841-7855. doi: 10.1021/ ie060309c

12. Taherkhani M, Tavakkoli-Moghaddam R, Seifbarghy M, Fattahi P. Detailed scheduling of tree-like pipeline networks with multiple refineries. International Journal of Engineering Transactions B Applications. 2017; 30(12): 1870-1878

13. Abbasi E, Garousi V. An MILP-based formulation for minimizing pumping energy costs of oil pipelines: beneficial to both the environment and pipeline companies. Energy Systems. 2010; 1(4): 393-416. doi: 10.1007/ s12667-010-0016-3

14. Zhang HR, Liang YT, Zhou XY, Yan XH, Qian C, Liao Q. Sensitivity analysis and optimal operation control for large-scale waterflooding pipeline network of oilfield. Journal of Petroleum Science and Engineering. 2017; 154: 38-48. doi: 10.1016/j.petrol.2017.04.019

15. Cafaro VG, Cafaro DC, Méndez CA, Cerdá J. MINLP model for the detailed scheduling of refined products pipelines with flow rate dependent pumping costs. Computers \& Chemical Engineering. 2015; 72: 210-221. doi: 10.1016/j.compchemeng.2014.05.012

16. Chen $H$, Wu C, Zuo L, et al. Optimization of Detailed Schedule for a Multiproduct Pipeline Using a Simulated Annealing Algorithm and Heuristic Rules. Ind. Eng. Chem. Res. 2017; 56(17): 5092-5106. doi: 10.1021/ acs.iecr.6b04745

17. Zhang $\mathrm{H}$, Liang $\mathrm{Y}$, Liao Q, Ma J, Yan X. An MILP approach for detailed scheduling of oil depots along a multi-product pipeline. Petroleum Science. 2017; 14(2): 434-458. doi: 10.1007/s12182-017-0151-8 
18. Mostafaei $\mathrm{H}$, Castro PM, Ghaffari-Hadigheh A. A Novel Monolithic MILP Framework for Lot-Sizing and Scheduling of Multiproduct Treelike Pipeline Networks. Ind. Eng. Chem. Res. 2015; 54(37): 9202-9221. doi: 10.1021/acs.iecr.5b01440

19. Polli HL, Magatão L, Magatão SNB, Neves F, Arruda LVR. Collaborative Approach Based on Heuristic Algorithm and MILP Model To Assignment and Sequencing of Oil Derivative Batches in Pipeline Networks. Ind. Eng. Chem. Res. 2017; 56(9): 2492-2514. doi: 10.1021/acs.iecr.6b03516

20. MirHassani SA, BeheshtiAsl N. A heuristic batch sequencing for multiproduct pipelines. Computers \& Chemical Engineering. 2013; 56: 5867. doi: 10.1016/j.compchemeng.2013.05.007

21. Relvas $\mathrm{S}$, Barbosa-Póvoa APFD, Matos HA. Heuristic batch sequencing on a multiproduct oil distribution system. Computers \& Chemical Engineering. 2009; 33(3): 712-730. doi: 10.1016/j.compchemeng.2008.10.012

22. Mostafaei $\mathrm{H}$, Castro PM. Continuous-time scheduling formulation for straight pipelines. Aiche Journal. 2017; 63(6). doi: 10.1002/aic.15563

23. Zhang $\mathrm{H}$, Liang $\mathrm{Y}$, Liao $\mathrm{Q}, \mathrm{Wu} \mathrm{M}, \mathrm{Yan} \mathrm{X}$. A hybrid computational approach for detailed scheduling of products in a pipeline with multiple pump stations. Energy. 2017; 119: 612-628. doi: 10.1016/j.energy.2016.11.027

24. Zhang $\mathrm{H}$, Liang $\mathrm{Y}$, Xiao Q, Wu M, Shao Q. Supply-based optimal scheduling of oil product pipelines. Petroleum Science. 2016; 13(2): 355-367. doi: 10.1007/s12182-016-0081-x

25. Relvas $\mathrm{S}$, Barbosa-Póvoa AP, Matos HA. Oil Products Distribution Systems: Decomposition Approach on Pipeline and Inventory Scheduling. Computer Aided Chemical Engineering. 2009; 27: 1971-1976. doi: 10.1016/ S1570-7946(09)70719-9

26. Zhang $H$, Liang $Y$, Liao Q, Gao J, Yan X, Zhang W. Mixed-time mixedinteger linear programming for optimal detailed scheduling of a crude oil port depot. Chemical Engineering Research and Design. 2018; 137: 434451. doi: 10.1016/j.cherd.2018.07.013

27. Relvas S, Matos HA, Barbosa-Póvoa APFD, Fialho J. Oil products pipeline scheduling with tank farm inventory management. Computer Aided Chemical Engineering. 2008; 25: 277-282. doi: 10.1016/S1570-7946(08)80051-X

28. Relvas S, Barbosa-Póvoa APFD, Matos HA. Inventory Management MILP Modeling for Tank Farm Systems. Computer Aided Chemical Engineering. 2010; 28: 727-732. doi: 10.1016/S1570-7946(10)28122-1

29. Cafaro VG, Cafaro DC, Méndez CA, Cerdá J. Optimization model for the detailed scheduling of multi-source pipelines. Computers \& Industrial Engineering. 2015; 88: 395-409. doi: 10.1016/j.cie.2015.07.022

30. Cafaro DC, Cerdá J. A Rigorous Mathematical Formulation for the Scheduling of Tree-Structure Pipeline Networks. Ind. Eng. Chem. Res. 2011; 50(9): 5064-5085. doi: 10.1021/ie101462k

31. Cafaro VG, Cafaro DC, Méndez CA, Cerdá J. Detailed Scheduling of Operations in Single-Source Refined Products Pipelines. Ind. Eng. Chem. Res. 2011; 50(10): 6240-6259. doi: 10.1021/ie200007a

32. Cafaro DC, Cerdá J. Optimal scheduling of multiproduct pipeline systems using a non-discrete MILP formulation. Computers \& Chemical Engineering. 2004; 28(10): 2053-2068. doi: 10.1016/j.compchemeng.2004.03.010

33. Relvas $S$, Boschetto Magatão SN, Barbosa-Póvoa APFD, Neves F. Integrated scheduling and inventory management of an oil products distribution system. Omega. 2013; 41(6): 955-968. doi: 10.1016/j. omega.2013.01.001

34. Relvas S, Barbosa-Póvoa APFD, Matos HA, Fialho J, Pinheiro AS. Pipeline scheduling and distribution centre management-A real-world scenario at CLC. Computer Aided Chemical Engineering. 2006; 21: 2135-2140. doi: 10.1016/S1570-7946(06)80364-0

35. MirHassani SA, Abbasi M, Moradi S. Operational scheduling of refined product pipeline with dual purpose depots. Applied Mathematical Modelling. 2013; 37(8): 5723-5742. doi: 10.1016/j.apm.2012.11.009
36. MirHassani SA, Fani Jahromi H. Scheduling multi-product tree-structure pipelines. Computers \& Chemical Engineering. 2011; 35(1): 165-176. doi: 10.1016/j.compchemeng.2010.03.018

37. Rejowski R, Pinto JM. A novel continuous time representation for the scheduling of pipeline systems with pumping yield rate constraints. Computers \& Chemical Engineering. 2008; 32(4-5): 1042-1066. doi: 10.1016/j.compchemeng.2007.06.021

38. Viswanathan J, Grossmann IE. A combined penalty function and outerapproximation method for MINLP optimization. Computers \& Chemical Engineering. 1990; 14(7): 769-782. doi: 10.1016/0098-1354(90)87085-4

39. Castro PM, Grossmann IE, Zhang Q. Expanding scope and computational challenges in process scheduling. Computers \& Chemical Engineering. 2018; 114: 14-42. doi: 10.1016/j.compchemeng.2018.01.020

40. Raman R, Grossmann IE. Modelling and computational techniques for logic based integer programming. Computers \& Chemical Engineering. 1994; 18(7): 563-578. doi: 10.1016/0098-1354(93)E0010-7

41. Castro PM, Grossmann IE. Generalized Disjunctive Programming as a Systematic Modeling Framework to Derive Scheduling Formulations. Ind. Eng. Chem. Res. 2012; 51(16): 5781-5792. doi: 10.1021/ie2030486

42. Castro PM, Barbosa-Póvoa AP, Novais AQ. Simultaneous Design and Scheduling of Multipurpose Plants Using Resource Task Network Based Continuous-Time Formulations. Ind. Eng. Chem. Res. 2005; 44(2): 343-357. doi: $10.1021 / \mathrm{ie} 049817 \mathrm{~h}$

43. Castro PM, Harjunkoski I, Grossmann IE. Expanding RTN discrete-time scheduling formulations to preemptive tasks. Computer Aided Chemical Engineering. 2018; 1225-1230. doi: 10.1016/B978-0-444-64241-7.50199-3

44. Castro PM, Barbosa-Póvoa AP, Matos HA, Novais AQ. Simple ContinuousTime Formulation for Short-Term Scheduling of Batch and Continuous Processes. Ind. Eng. Chem. Res. 2004; 43(1): 105-118. doi: 10.1021/ ie0302995

45. Castro PM. Optimal Scheduling of Pipeline Systems with a Resource-Task Network Continuous-Time Formulation. Ind. Eng. Chem. Res. 2010; 49(22): 11491-11505. doi: 10.1021/ie1010993

46. Relvas $\mathrm{S}$, Matos HA, Barbosa-Póvoa APFD, Fialho J. Reactive Scheduling Framework for a Multiproduct Pipeline with Inventory Management. Ind. Eng. Chem. Res. 2007; 46(17): 5659-5672. doi: 10.1021/ie070214q

47. Balasubramanian J, Grossmann IE. Scheduling multistage flowshops with parallel units-An alternative approach to optimization under uncertainty. Computer Aided Chemical Engineering. 2003; 154-159.

48. Ryu JH, Dua V, Pistikopoulos EN. Proactive Scheduling under Uncertainty: A Parametric Optimization Approach. Ind. Eng. Chem. Res. 2007; 46(24): 8044-8049. doi: 10.1021/ie070018j

49. Li Z, lerapetritou M. Process scheduling under uncertainty: Review and challenges. Computers \& Chemical Engineering. 2008; 32(4-5): 715-727. doi: 10.1016/j.compchemeng.2007.03.001

50. Honkomp SJ, Mockus L, Reklaitis GV. A framework for schedule evaluation with processing uncertainty. Computers \& Chemical Engineering. 1999; 23(4-5): 595-609. doi: 10.1016/\$0098-1354(98)00296-8

51. Lin $\mathrm{X}$, Janak SL, Floudas CA. A new robust optimization approach for scheduling under uncertainty:: I. Bounded uncertainty. Computers \& Chemical Engineering. 2004; 28(6-7): 1069-1085. doi: 10.1016/j. compchemeng.2003.09.020

52. Cui J, Engell S. Medium-term planning of a multiproduct batch plant under evolving multi-period multi-uncertainty by means of a moving horizon strategy. Computers \& Chemical Engineering. 2010; 34(5): 598619. doi: $10.1016 /$ j.compchemeng.2010.01.013

53. Sand G, Engell S. Modeling and solving real-time scheduling problems by stochastic integer programming. Computers \& Chemical Engineering. 2004; 28(6): 1087-1103. doi: 10.1016/j.compchemeng.2003.09.009 